\title{
Virtual Articulator - Aid Simulator at Diagnosis, Pre-Surgical Planning and Monitoring of Bucomaxilofacial Treatment
}

\author{
Marta Becker Villamil ${ }^{\mathrm{a}}$ and Eduardo Garcia ${ }^{\mathrm{b}}$ \\ Universidade do Vale do Rio dos Sinos \\ Sao Leopoldo, RS - Brazil \\ ambvillamil@unisinos.br, ${ }^{b}$ EduardoGarcia@gmail.com
}

\begin{abstract}
This work presents a system for use in dentistry and medicine, that allows advance in diagnosis and planning of treatments and surgical procedures, in cases that involves the Temporomandibular Joint, TMJ. Construction of Virtual Articulator includes related research areas of computer graphics, virtual reality and medicine and tends to become a new paradigm as a tool because it will simulate and reproduce the movements of the TMJ in a realistic way, allowing a complete analysis of the case under treatment. It is a software which comes to replace and increase in an innovative way the work done by mechanical articulators. Initially Virtual Articulator reconstructs the TMJ virtually, generating a 3D model, starting from exams such as Computed Tomography and Magnetic Ressonance. Once it is obtained a virtual copy of the TMJ, software simulates real mandible movements, with great flexibility and facility of parameterization. Virtual joints model is based on points captured from the motion curve of lower incisor point. Contribution of each muscle in temporomandibular movement is approached from Hill actuators model and the new concept of curves of insertion. It will be possible to analyze in depth a particular case in a diagnostic phase or predict the results of the surgical procedure.
\end{abstract}

\section{Introduction}

Among the articulations systems presented in human body, the masticatory system provides many aspects for investigation. Simulation of the structural and functional elements that work as a group is specially useful when related to cause and effect. Contractions of many muscles of different shapes and sizes guided by two joints make kinematic and physical of this system not fully understood [2]. Jaw motion simulators build to education in destistry, orthodontic adjustment of occlusions, or preoperative planning of craniofacial surgery can be extremely useful, improving diagnosis and postoperative treatment. Relationship between muscle actions and mandibular movement is probably the least understood aspect of mandibular biomechanical, despite its important meaning. Jaw is a moving object according to dynamic laws. It has attributes such as mass, center of mass and inertial properties that are not generally described.

Virtual articulators already proposed in past works include only the mandibular function based on motion captured by devices attached to the lower teeth [17] [18] [19] or simulate the unrealistic movements of a mechanical articulator [20].

Works as Stavness [6] are already being developed in order to simulate the effects of mandible reconstruction. Treatment of cancer patients often require the removal of part of bone and muscle structure, changing facial anatomy and function of the muscles. These changes usually require an esthetics and functional reconstruction of the mandible. A preoperative simulation system may help experts to plan procedures increasing its analysis. Stavness [6] work uses a simulation platform, called Artisynth, based on rigid body simulation, collision detection algorithms and muscle actuators dynamic non-linear Hill type [1]. Simulation control is done through an activation level curve versus timeline, for each muscle.

Our work proposes a system for use and studies in dentistry and maxillofacial surgery. The main contribution is to simulate masticatory system as a whole, including muscles contribution and ligaments restrictions in the mandibular movement. In addition it presents an in-depth study of movement generated by two TMJs interconnected.

\section{Materials and Methods}

Models that simulate chewing process can be developed in three basic steps: the first one is in vivo medical imaging and $3 \mathrm{D}$ reconstruction of anatomic parts. Second step is to establish joints center of 
rotation and build a model that deal with the interdependence of left and right TMJ. Another challenge is to model muscles, tendons and ligaments as a complex. Finally, it's necessary to simulate muscle contraction, resulting in a trajectory that can now be validated by comparison with experimental data.

\subsubsection{D model reconstruction}

Acquisition. Data was acquired on a PQ5000 CT scanner (Marconi Medical Systems, Cleveland, Ohio) with $1.0 \mathrm{~mm}$ slice spacing and thickness, $24.0 \mathrm{~cm}$ FOV. Images are $512 \times 512$ pixels, each pixel representing $0.4687 \mathrm{~mm}$. The total set contains 252 axial slices (12 bits/pixel grayscale information) in DICOM format. The acquisition protocol was chosen from a preset protocol menu $(120 \mathrm{kVp}, 100 \mathrm{~mA}, 15.7$ seconds). To facilitate the distinction of structures during the reconstruction procedure and avoid the teeth occlusion, the volunteer's mouth was kept open by means of three LEGO ${ }^{\circledR}$ blocks (around $3 \mathrm{~cm}$ ) between her mandible and superior teeth allowing to obtain better teeth shape accuracy.

Reconstruction. In order to reproduce and visualize the mastication movement, we need the mandible as a separate object from the rest of the head. The 3D model was planned as three triangular meshes, one for the mandible, and the other two for the superior teeth and the cranium, respectively. To obtain these meshes, firstly a segmentation process was carried out, and then a mesh extraction technique was selectively applied. The segmentation and surface model generation were performed using the program SLICER [21].

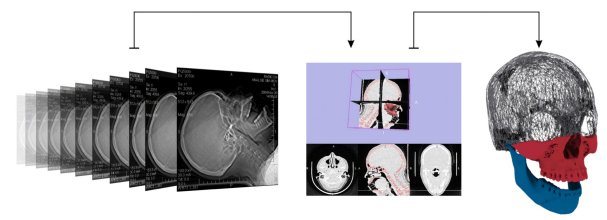

Figure 1: From the computed tomography images, region of the bones and teeth were segmented for obtaining the triangle meshes.

The method we followed for separating the regions is summarized below. All the steps were accomplished with interactive functions provided by the SLICER program:
1. The slices were segmented using a threshold interval of $[1,411 ; 4,095]$ for separating bones and teeth. The pixels in this interval were assigned red color

2. To separate the superior teeth region from the rest of the cranium, we converted the pixels of two slices back to the original grayscale values. The slices were selected based on a location adequate to separate the desired region. The pixels in this region were assigned magenta values.

3. The pixels corresponding to the mandible are well separated from the rest of the cranium. They were assigned yellowish color.

4. The cranium bones were isolated from the brain region by means of successive interaction using cutting planes and color assignment. The final bone region was assigned blue color.

5. The Marching Cubes algorithm available in SLICER was used to selectively extract the three 3D meshes, each one identified by a specific color (Figure 2).

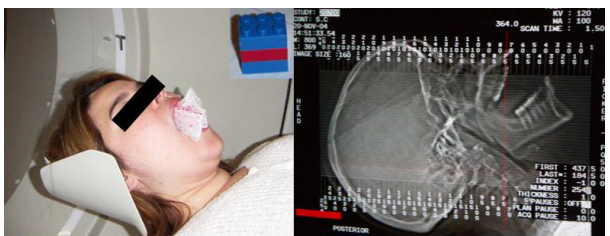

Figure 2: The volunteer kept the mouth opened during the tomography.

Typically, the surface model generated by the Marching Cubes algorithm is highly detailed but unfortunately composed by millions of triangles. The decimation algorithm, also available in SLICER, was used as a polygon reduction technique yet preserving the original topology and forming a good approximation of the original geometry. Due to the need of high resolution for dealing with collision between teeth during simulation, the mandible and superior teeth meshes have higher accuracy than the mesh of cranium bones. They have 97, 105 and 115 thousands triangles, respectively.

First step in building a virtual human model consists on acquiring and processing images, used to three-dimensional reconstruction of the human body parts. Since one of the main reasons for simulating human masticatory system is to understand the process itself, models must be based on data which is as solid as possible, by data imaging with MRI or CT scan of muscle, joints and teeth. Three-dimensional 
representations can be obtained from pixel-based on two-dimensional slices or three-dimensional arrays, both provided by an imager or by using a 3D scanner in as plaster cast of a real arcade. We used, in our model, CT images scanned from a healthy volunteer [24] and [25]. Scanned model is separated into three different meshes of polygons: one to the jaw, one representing the upper teeth, and another representing the skull. Skull was scanned separately from the upper jaw because it requires a lower resolution and less computational resources, but final result was the same (Figure 1).

A contact surface to slide the condyle was added. Use of condylar surfaces simulating the cavity has been previously employed by Hannan et al. [12] and [7. In our work, however, surface is being used as an upper contact limit, in order to better simulate function of bone structure, cartilage (articular disc) and other tissues. Shape of surface was defined by points that formed a Spline surface. Curve parameters were partially based on experimental data with healthy patients and previous published studies. Small adjustments were made at each simulation.

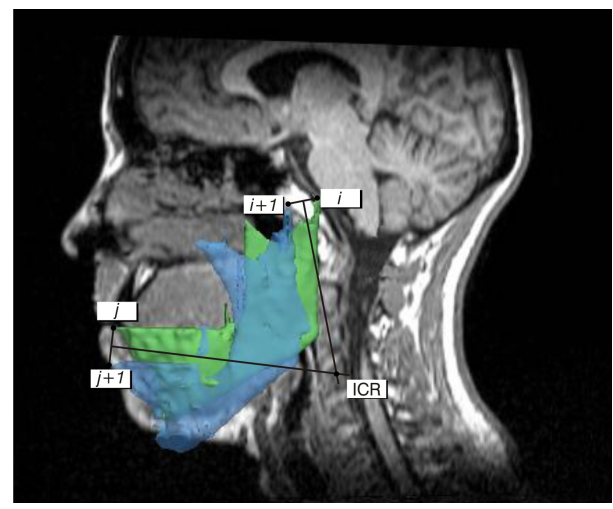

Figure 3: Representation of the calculation of the initial rotation center of TMJs or origin of the vector base representing the TMJ.

\subsection{Initial Rotation Center Positioning}

Method known as the center of instantaneous rotation [3], is frequently used in mechanical analysis of joints to find the center of rotation when the movement is described only by rotations. Jaw movement is described as rotational only in the first millimeters of mouth opening movement. That is why only first rotation center is calculated by using this method.
After, origin and direction of next rotation axes are obtained from path described by incisal point and treatment of mandible and maxilla collisions.

Initial rotation centers (IRC), for the TMJs left and right, are obtained through calculations using anatomical points marked in Magnetic Resonance Images. Jaw area is segmented into images in different positions. First, mouth totally closed and other with mouth partially open with one centimeter between upper and lower incisors (Figure 3).

Three anatomical points were located at the top of the condyles (i) and in the center between the lower incisors (j). For each joint was considered the motion of these two points (each condyle and incisal) $\left(i^{t} ; j^{t}\right)$ for the time period $t$ for $+1\left(i^{t}+1 ; j^{t}+1\right)$ Center of rotation for the movement increment can be calculated raising perpendicular bisectors between lines $\left(i^{t}\right)$ to $\left(i^{t}+1\right)$ and $\left(j^{t}\right)$ to $\left(j^{t}+1\right)$. The center of rotation is between the intersection of two bisectors (Figure 3).

Temporomandibular joint model [7], [8] consists in a pair of joints with three rotational and three translational DOFS (Degrees of Freedom) each one, that moves a single bone, mandible. Movements restrictions are in function of collision between bones and teeth, and by a topology of joints (motion generated by a joint interferes in the positioning of the adjacent joint). Each joint has its own reference frames, but its behavior in terms of rotation and translation is also dependent of the adjacent joint. TMJ reference frames are in charge of the position and orientation of the polygon mesh that represents jaw. TMJs motion model is based on a sequence of positions and orientations of two references frames that represent the left and right TMJs. This sequence was inferred from the incisal path captured from an individual with normal TMJs. Transformations to perform movement are obtained by inverse kinematics, collisions treatment and from restrictions imposed by interdependent topology which defines the relationship between the movement of the left and right TMJs.

Having obtained the ICRs that determine the starting point of each condylar path, the rest of this path is obtained from inverse kinematics and collision avoidance.

For inverse kinematics, the incisal point is set as end-effector, and each sample point along the Catmull-Rom spline is set as a goal position. The linkage is simple enough for directly calculating joint angles by using an analytical approach as there is only one rigid segment (mandible) connected to TM joints. Cross product between the vector originated at TMJ frame center to the incisal point and the vector from the same origin to the captured point gives the joint axis of rotation (Figure 4). The angle of rotation is 
obtained by dot product between these vectors. First of all, mandible rotation tries to reach the goal position. If this is not sufficient, a mandible translation is performed. Translation moves both TMJ's origins and the mandible graphic object. The normal condylar path corresponds to this sequence of transformations. Since the model was derived from a specific normal subject, it would not be valid without fitting to different bones and teeth morphologies. Next section presents the generalization of our TMJ motion model.

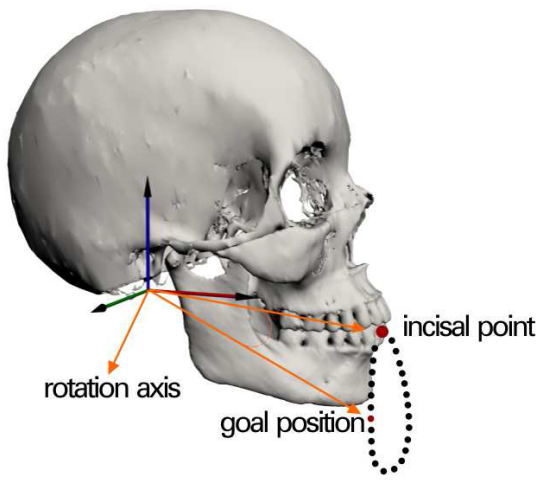

Figure 4: Two vectors used to calculate angle and axis of rotation. They are originated at TMJ frame. One points to the incisal point and the other one points to a position along the captured path.

The normal condylar path is represented by the sequence of transformations that should be applied to $T M J_{\text {left }}$ and $T M J_{\text {right }}$ to move the mandible. Applying the same sequence of transformations to different bones morphology for sure would result in collision between the jaw and temporal bones in the condylar socket.

So, the model is completed by a collision detection and treatment method, to avoid interpenetration of meshes representing the bones of the cranium and the mandible. The collision detection module uses Oriented Bounding Boxes hierarchies [26] to check, at each motion iteration, if there is a collision Once a collision is detected, when a rotation is performed, the origin of the reference frames that represent the TMJs should be moved to a point closer to the joint. The cause of this adjustment is that the closer the origin of the rotation axes is to the condyle center, less the condyle will dislocate inside the condylar socket (Figure 5). This adjustment is accomplished through a sequence of small steps of reference frames displacements until the rotation does not cause collision anymore.

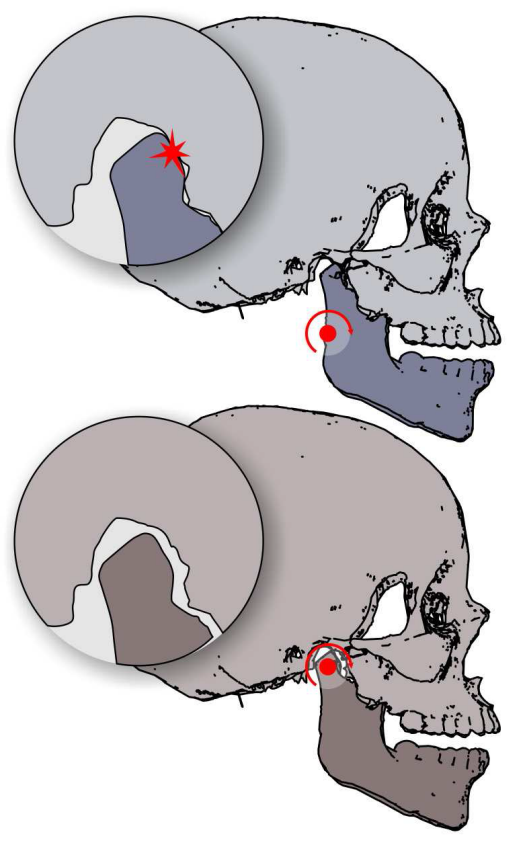

Figure 5: If the origin of rotation axis is closer to the condyle center, there will be less collisions between the mandible bone and the condylar socket.

Otherwise, if a collision is detected during a mandible translation, the algorithm will determine iteratively a different displacement. At first, a shorter translation is tried along the same direction. If a collision is still detected, the algorithm adjusts the origin of the translation in the sagittal plane based on the current TMJ origin and on the collision point (Figure 6). 


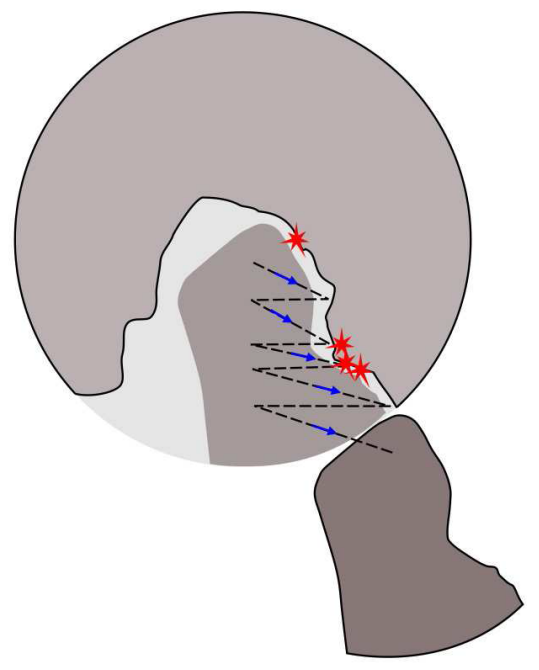

Figure 6: The algorithm tries to find another path to the mandible based on the saved path and on collision avoidance.

\subsection{Interrelation of Muscle Forces Model}

Complementation of model based on kinematics with one model based in Physics that simplifies the muscles contribution over the jaw through lines of action will lead to a complete understanding of the movement of the masticatory system.

Muscle forces requires modelling a physical simulator of TMJ. It allows to change parameters according to the model adopted. PhysX physics software [16] analyzes the forces and constraints applied to mandible, identifies and respond to collisions between superior and incisor arcs, and updates the condition of variables. Simulation results in the real time response visualization through a rendered model and the generation of trajectories from the incisal point which can be compared with real jaw trajectories.

Muscles were modeled by curves representing the origin and insertion of its fibers using Hill-type effectors [1]. During simulation, which takes place in real time, user can change level of activation of each muscle group, observing the reaction of the model to the set of forces exerted by muscles. Trajectories were determined by forces influence derived from muscle contraction, which were based on the levels of muscle activation and on various parameters that were adjusted for each muscle of the complex.

Muscles were set manually, in accordance with the literature and the magnetic resonance images obtained from one volunteer, who served as a reference for a correct definition of these points. Use of muscle straight lines is commonly performed to estimate the action of their activation of the structure of bones. This straight line is usually set over two main points: the point of origin which is usually inserted in a fix region, and an insertion point associated with the bone that moves. We took this design as a reference but, instead of insertion points, we used the concept of origin and insertion curves. Fact that TMJ muscles exhibit a much more rectangular than long profile, when compared to other muscles of the human body such as the upper and lower limbs, was our motivation to adapt this suggestion. In doing so, we hoped to obtain more realistic results.

Here, six groups of muscle were modeled. Anterior temporal and temporal muscles, followed by the masseter, superior lateral, inferior lateral and medial pterygoid. Right side of the jaw was also modeled, and the highest possible symmetry between the two sides was sought.

Activation curves simulate how nerve stimulation generates the basic movements of the jaw. Data are entered graphically through the activation contours ranging from 0 to 1 ( 0 to 100$)$ as a function of time. Data on the levels of activation were investigated by Moller [14], Hannan and Wood [9] and Hiraba et al. [15], among others (Figure 7).
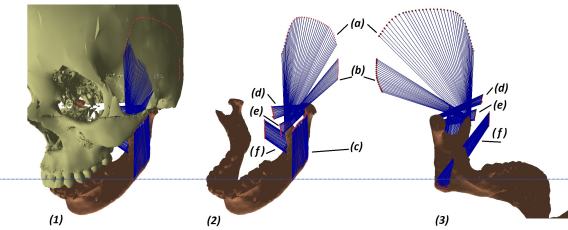

Figure 7: Definition and arrangement of the origin and insertion curves for temporal (a), anterior temporal (b), masseter (c) and superior lateral (d), bottom (e) and medial (f) pterygoid muscles, seen in the full model (1) and in the mandible external (2) and internal (3) views.

Therefore, Hill model continues to be widely used in biomechanical simulations. Despite its wellknown limitations [10], such as its inability to provide information about the internal structure of muscles, it is still largely employed due to its low computational demand and realistic results, as well as for its ability to produce torque in the generation of realistic 
movements. Hill model was used for calculating the force generated for each muscle fiber at each step of simulation. By isolating the tension $T$, we have:

$$
T=\frac{\left(K_{P E}\left(x-x^{*}\right)+b\left(\dot{\mathrm{x}}-\frac{\dot{\mathrm{T}}}{K_{S E}}\right)+A\right) K_{S E}}{K_{S E}+K_{P E}}
$$

Where $T$ is tension $K_{P E}$ and $K_{S E}$ are the elastics elements in parallel and series, respectively. $\dot{\mathrm{x}}$ is the velocity and $A$ is the muscle active force.

Values for speed of contraction $\dot{x}$ and rate of change of tension $\dot{T}$ get closer and closer to each other at each iteration of simulation. Damping coefficient $b$ represents the force-velocity relationship in a linear fashion. In addition, when using the Hill model the tension-length ratio must always be followed. Ways in which these relations have been simulated are detailed below.

There is a well-known connection between the length of a muscle and the maximum active tension that it can develop. This is caused by the level of tension to the muscle and the associated number of junctions that form cross bridges in each sarcomere of the muscular tissue. When a muscle changes its length there is a proportional decrease in the amount of overlap between them and, therefore, a reduced amoun of tension that the sarcomere can develop [11]. In this work, this relationship has been simplified following the work of Cofer et al. [12], who used the following equation:

$$
T(x)=1-\frac{\left(x-x^{*}\right)^{2}}{x_{l}^{2}}
$$

where $x^{*}$ is the length of the muscle at rest and $x_{l}$ determines how the generated force will be attenuated by muscle contraction or extension. Increase in $x_{l}$ enhances the distribution of the inverse quadratic function resulting in a lower attenuation levels of force.

In this work, however, a linear approximation was used instead of original hyperbolic equation. This type of approach is called Hill Linear Muscle [12], and was the one used in the present study. This is the reason why the insertion of a damping coefficient, $b$, was required in the muscle model.

Four ligaments were modeled: initially the capsular ligament, which has the function to limit the movement of the inferior and posterior condyle. Next, the posterior ligament was included, limiting the movement of the condyle protrusive. Then the temporomandibular ligament was added, whose function is limiting lateral movements. Finally, the stylomandibular ligament was considered which limits the protrusive movement of the jaw. We chose not to model the ligament sphenomandibular, since this is an accessory ligament and not limited any movement of the jaws.

\section{Results}

Posselt figure [28] is the maximum area that encompasses all the points that can be achieved by incisal point (point between lower incisors). It has been considered the typical mandible motion curve viewed at sagittal plane. We compared our motion curve simulated with the real one recorded by Wilson and Banerjee [27].

In our previous work [23], the trajectory described by the jaw during basic movements such as opening, closing and chewing was captured using a magnetic sensor. This sensor allowed the acquiring of a threedimensional position set next to the incisors of a volunteer, which also had his skull scanned in a CT.

After the simulation process, the trajectory described by the control point was compared to the real path of the jaw observed with the use of the scanner. Small adjustments were made in the activation curves of muscles in order to t the model to the real movement of the jaw. However, the use of the scanner does not provide information concerning the force of individual muscle during the trajectory, and therefore a complete validation of the model was not possible. Even though, it is expected that forces generated by different muscles are within normal limits since the contribution of their maximum strength was chosen based on previously published studies [22].

Comparison of the Opening and Closing Movements. Figures 8 and 9 illustrate the data for the simulation of the jaw opening and closing and comparison with data acquired from a volunteer by motion capture. Fifteen cycles of opening and closing were performed during both simulation and real data collection.

Comparison of chewing movements. Figure 10 present data for the simulation of the mastication motion and comparison with data acquired from a volunteer. Fifteen cycles of opening and closing were performed during both simulation and real data collection. 


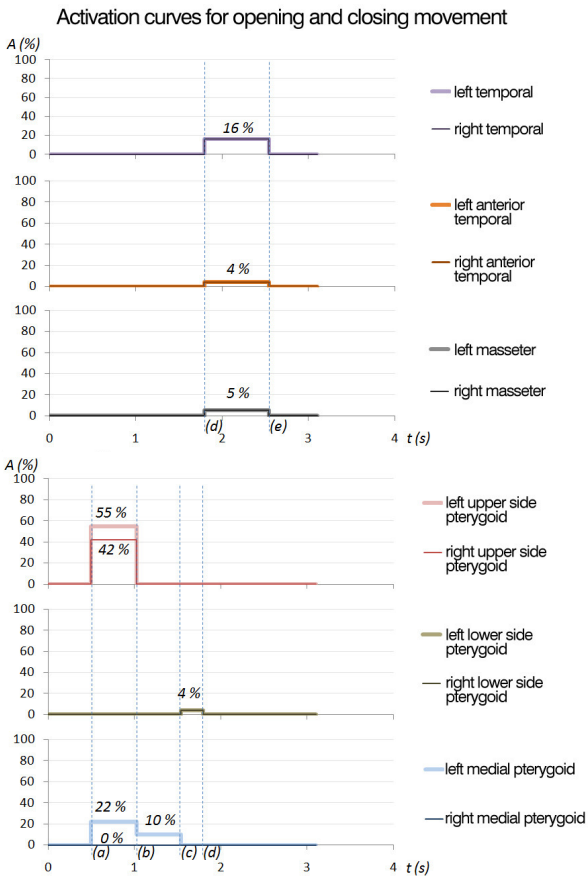

Figure 8: Activation curves to obtain jaw opening and closing trajectories. Beginning with the opening action of the superior lateral pterygoid (a) motion with right medial pterygoid (c) and closing with the muscles of the temporal, masseter and lower lateral pterygoid (d).

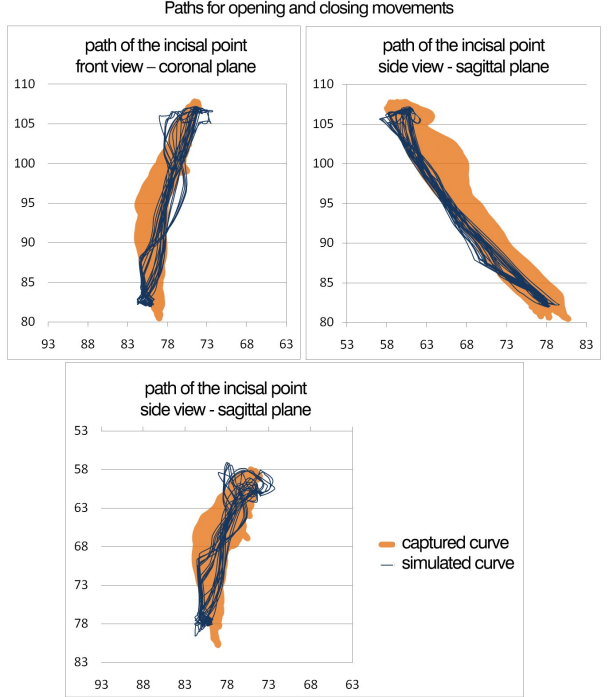

Figure 9: Comparison between convex hull of the trajectory captured and the simulated trajectory, both in 15 cycles of jaw opening and closing.

Dimensions in $\mathrm{mm}$. 


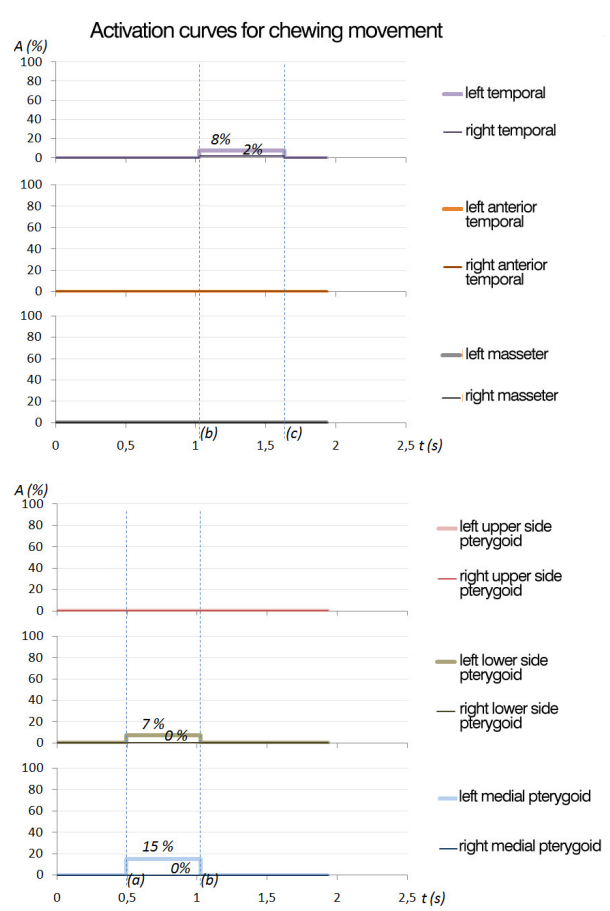

Figure 10: Activation curves to obtain the mastication trajectory.

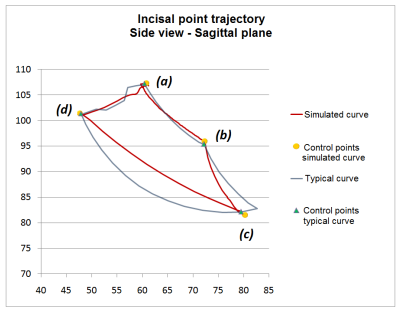

Figure 11: Mandible motion curve obtained compared with the one recorded by Wilson and Banerjee [27]. Error measurements at control points are measured in $\mathbf{~ m m}$.

\section{Conclusion}

In this paper we introduced a Virtual Articulator to simulate human temporomandibular joint behavior for medical purposes. The model was calibrated using images obtained from CT and MRI, and a motion path captured by a motion capture system. The Virtual Articulator is based on joints topology which deals with the balanced motion between right and left TMJs. The internal joint motion path was derived from the external motion path generated by the opening/closing mandible movements previously tracked by using the proposed joint model, inverse kinematics and collision avoidance. Muscles were modeled by curves representing the origin and insertion of its fibers using Hill-type effectors.

Validation is accomplished by comparing the real articulation movement acquired with the ones generated by us through image registration process and dynamic MRI images. Model validation requires that the modeled trajectories reproduce those of the jaw of the volunteer who provided the data. However, the trajectory of the moving jaw of the volunteer is not stable or reproducible. More- over, it is important to underline that the control of movements is performed by the brain, which works almost automatically in accordance to our learned neural coordinate muscle movements. Model has no feedback system for automatic correction of the position, so differences between the volunteer trajectory captured and the trajectory obtained by simulation are expected. Demonstrations that simulated movements are running a similar course and that simulated trajectories remain within a convex region formed by the acquired trajectories of the volunteer can be considered an acceptable validation. As future works we also intend to model motion of others mandible anatomies like Prognathism. In opposite of retrusion, prognathism is the positional relationship of the mandible and/or maxilla to the skeletal base where either of the jaws protrudes beyond a predetermined imaginary line in the coronal plane of the skull.

We also intend to simulate small mastication movement patterns and analyze how they change based on teeth and food collision.

\section{Acknowledgments}

The authors would like to thank their collaborators from the Department of Oral and Maxillofacial Surgery, Cliniques Universitaires Saint Luc, Université catholique de Louvain (UCL), Belgium. In particular, we are deeply grateful to Dr. Raphael Olszewski from UCL for fruitful discussions along this work, and Prof. Marcio Lima Grossi from the Post-Graduate Program in Dentistry of the Pontifical Catholic University of Rio Grande do Sul, Brazil, for helping with data 
acquisition. We are also grateful to the three volunteers that accepted to help us by providing the input data (CTs, MRIs, motion data, and cephalometric measures) for this research.

\section{References}

[1] Hill, A. The heat of shortening and the dynamic constants in muscle. Proc.Roy.Soc.London, v. 126, p. 136 195, 1938.

[2] Lemoine, J. J.; Xia, J. J.; Gateno, J.; Liebschner, M. A. K. Radiographic Analysis for Jaw Motion Normalization. Journal of Oral Maxillofacial Surgery, [S.1.], v.63, p.961967, 2005

[3] Lepera, F. Determination of the hinge axis clutches on condyle position. Journal of Prosthetic Dentistry, [S.1.], v.8, p.260, 1958.McNeill C. Ciência e Prática da Oclusão. 1 ed, Ed. Quintessence, São Paulo, 2000.

[4] SLICER software (2005). http://www.slicer.org/

[5] Stavness, I. et al. An integrated, dynamic jaw and laryngeal model constructed from CT data. In: Proc ISBMS06 in Springer LNCS 4072. [S.1.]: Springer, 2006. p.169 177

[6] Stavness, I. et al. Towards predicting biomechanical consequences of jaw reconstruction. Conf Proc IEEE Eng Med Biol Soc, v. 2008, p. 4567 4570, 2008

[14] E. Moller, The chewing apparatus. an electromyographic study of the action of the muscles of mastication and its correlation to facial morphology., Acta Physiol Scand Suppl 280 .

[15] K. Hiraba, K. Hibino, K. Hiranuma, T. Negoro, Em activities of two heads of the human lateral pterygoid muscle in relation to mandibular condyle movement and biting force., J Neurophysiol 83 (4) (2000) 2120-2137.

[16] PhysX software

http://www.nvidia.com/object/physx faq.html

(2016)

[17] A. Bisler, U. Bockholt, G. Voss. The virtual articulatorapplying VR technologies to dentistry. Information Visualization, 2002. Proceedings. Sixth International Conference on IEEE, 2002.

[18] C. Gärtner, B. Kordass. The virtual articulator: development and evaluation. International Journal of Computerized dentistry 6.1 (2003): 11 .

[19] E. Solaberrieta, et al. Design of a virtual articulator for the simulation and analysis of mandibular movements in dental CAD/CAM. Proceedings of the 19th CIRP Design Conference-Competetive Design. Cranfield University Press, 2009.
[7] R. Olszewski , ; Villamil, m. b. ; Trevisan, d. g. ; Nedel, 1. p. ; Freitas, c. d. s. ; Reychler H., ; Macq, B. . Towards an integrated system for planning and assisting maxillofacial orthognathic surgery.. Computer Methods and Programs in Biomedicine (Print), v. 91, p. 13-21, 2008

[8] M. B. Villamil, L. P. Nedel, C. M. Freitas, B. Macq, Simulation of the human tmj behavior based on interdependent joints topology, Computer Methods and Programs in Biomedicine. , v. 105(3), p. 217-232, 2012

[9] A. Hannan, W. Wood, Medial pterygoid activity during the closing and compressive phases of human mastication. American Journal of Physical Anthropology 55 (1981) 359367.

[10] Y. Fung, Biomechanics: Mechanical Properties of Living Tissues, Springer, 1993

[11] H. E. Huxley, M. Kress, Crossbridge behaviour during muscle contraction, Journal of Muscle Research and Cell Motility 6 (2) (1985) 153-161.

[12] D. Cofer, G. Cymbalyuk, J. Reid, Y. Zhu, W. J. Heitler, D. H. Edwards, Animatlab: a 3d graphics environment for neuromechanical simulations., J Neurosci Methods 187 (2) (2010) 280-288

[13] J. M. Winters, S. L. Y. Woo, Multiple Muscle Systems. Biomechanics and Movement Organization, Springer, New York, US-NY, 1990.

[14] E. Moller, The chewing apparatus. an electromyographic study of the action of the muscles of mastication and its correlation to facial morphology., Acta Physiol Scand Suppl

20] Y. Otake, N. Suzuki, A. Hattori, Y. Shigeta, T. Ogawa, S. Fukushima, A. Uchiyama. (2006). Real-time mandibular movement analysis system using fourdimensional cranial bone model. System and COmputers in Japan, 37(8), 1-12.

[21] Slicer software (2016). https://www.slicer.org/

22] G. E. Langenbach, A. G. Hannam, The role of passive muscle tensions in a threedimensional dynamic model of the human jaw., Arch Oral Biol 44 (7) (1999) 557-573

[23] M. B. Villamil, L. P. Nedel, C. M. Freitas, B. Macq, Simulation of the human tmj behavior based on interdependent joints topology, Computer Methods and Programs in Biomedicine. doi:10.1016/j.cmpb.2011.09.010

[24] M.B. Villamil, L.P. Nedel, C. Freitas, A. Maciel, A model to simulate the mastication motion at the temporomandibular joint, in: SPIE MEDICAL IMAGING 2005-Physiology, Function, and Structure from Medical Images, vol. 6, San Diego, USA, 2005.

[25] M. B. Villamil, L. P. Nedel, C. M. Freitas, B. Macq (2012). Simulation of the human TMJ behavior based on 
interdependent joints topology. Computer methods and programs in biomedicine, 105(3), 217-232.

[26] S. Gottschalk, M. C. Lin, D. Manocha, Oobtree: A hierarchical structure for rapid interface detection, in: Proceedings of SIGGRAPH, 1996, pp. 171-180.
[27] WILSON, P. H. R.; BANERJEE, A. Recording the retruded contact position: a review of clinical techniques. British Dental Journal, v. 196, p. 395-402, 2004.

[28] Studies in the mobility of the human mandible Salzmann, J.A. American Journal of Orthodontics , Volume 39 , Issue $6,471-473,1953$ 